\title{
Design of Vertically Stacked Waveguide Filters in LTCC
}

\author{
Tze-Min Shen, Chi-Feng Chen, Ting-Yi Huang, and Ruey-Beei Wu, Senior Member, IEEE
}

\begin{abstract}
This paper proposes four-pole quasi-elliptic function bandpass waveguide filters using multilayer low-temperature co-fired ceramic technology. The vertical metal walls of the waveguide resonators are realized by closely spaced metallic vias. Adjacent cavities are coupled by a narrow slot at the edge of the common broad wall or an inductive window on the sidewall. Two types of vertical coupling structures are utilized to achieve the cross coupling between nonadjacent resonators at different layers. With multilayer capability, there is more flexibility to arrange the cavities of coupled resonator filters in 3-D space. It is demonstrated by both the simulation and experiment that the proposed filter structures occupy a compact circuit area and have good selectivity. The filter with electric field cross coupling occupies a half area of a planar four-pole waveguide filter, while the filter with stacked vias cross coupling has $65 \%$ size reduction in comparison with a planar waveguide filter.
\end{abstract}

Index Terms-Bandpass filter, cavity, coupling coefficient, lowtemperature co-fired ceramic (LTCC), stacked vias, quasi-elliptic function.

\section{INTRODUCTION}

$\mathbf{M}$ ODERN microwave communication systems require high-performance bandpass filters with high selectivity, low insertion loss, and compact size. Filters with a waveguide structure can offer low loss and a high quality $(Q)$ factor, but usually at the price of large size, heavy weight, and high cost. The manufacturing of the waveguide also needs sufficient accuracy in order to operate at the millimeter-wave frequency. Recently, the concept of synthesized rectangular waveguide structures [1] has attracted much interest. The waveguide is dielectric filled and embedded into a substrate. The sidewall of the rectangular waveguide can be realized by arrays of metallic via or metallic grooves. This kind of waveguide not only maintains a good $Q$ factor, but also suits the realization of high-performance bandpass filters at the millimeter-wave frequency regime.

Several direct-coupled cavity filters have been realized by the synthesized waveguide structures on flip-chip modules [2], printed circuit board [3], and thick-film technology [4]. These filters usually occupy a large circuit area because of the planar arrangement of the resonators. With the mature multilayer technology, synthesized waveguide filters are also fabricated on a

Manuscript received February 6, 2007; revised May 10, 2007. This work was supported in part by the National Science Council, Taiwan, R.O.C., under Grant NSC 93-2752-E-002-003-PAE and Grant NSC 94-2219-E-002-001 and by the Industrial Technology Research Institute.

The authors are with the Department of Electrical Engineering and Graduate Institute Communication Engineering, National Taiwan University, Taipei, 10617 Taiwan, R.O.C. (e-mail: rbwu@ew.ee.ntu.edu.tw).

Digital Object Identifier 10.1109/TMTT.2007.902080 low-temperature co-fired ceramic (LTCC) technology [5] and micromachined process [6]. Multilayer filter technology provides significant benefits in terms of design flexibility and density. This makes vertical coupling between resonators possible and cavities can be piled up in 3-D space, which will largely reduce the circuit area.

Frequency selectivity is also an essential feature of a highperformance filter. Quasi-elliptic or elliptic filters will have transmission zeros at finite frequencies and give more improved stopband rejection than conventional direct-coupled filters [7]-[9]. Such filter responses can be realized with cross coupling between nonadjacent resonators [10]. The zeros are then obtained by means of destructive interference between the different signal path connecting the input and output ports [11] Recently, a conventional parallel coupled microstrip filter with a transmission line inserted inverter for realization of different advanced filtering characteristics was presented in [12]. With the additional cross-coupled transmission line, there is greater flexibility in the arrangement of the cross coupling path to achieve the desired frequency response.

In this paper, quasi-elliptic bandpass filters with a cross-coupling architecture are developed in the multilayered LTCC technology, as shown in Figs. 1 and 2. An open-ended microstrip line is used to excite the filters by a narrow slot etched on the first/last cavity. The LTCC resonators can be stacked three-dimensionally to provide various coupling mechanisms required in the design of quasi-elliptic bandpass filters, while achieving compact sizes and good selectivity. The cross coupling between nonadjacent resonators is achieved by a square aperture at the center of the common wall in Fig. 1 or by additional stacked vias and short-circuited coplanar waveguides (CPWs) in Fig. 2.

This paper is organized as follow. Section II describes the key design parameters required to realize the quasi-elliptic filter. Section III introduces several coupling structures and the relation of coupling coefficients versus physical dimensions. Section IV provides two design examples. The experiment data are presented and compared with simulation results. Finally, some brief conclusions are drawn in Section V.

\section{FILTER DesigN}

A general coupling structure of a quasi-elliptic filter is depicted in Fig. 3 [10], where each node represents a resonator, and the solid and dashed lines indicate the main and cross-coupling paths, respectively. It is essential that the signs of the coupling coefficients $M_{m-1, m+2}$ and $M_{m, m+1}$ are opposite in order to realize a pair of attenuation poles at the finite frequencies. This means that the coupling routes of $M_{m-1, m+2}$ and $M_{m, m+1}$ 


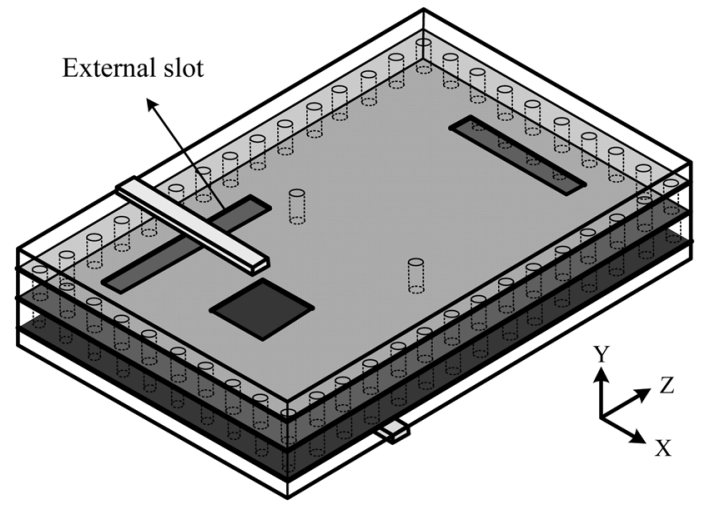

(a)

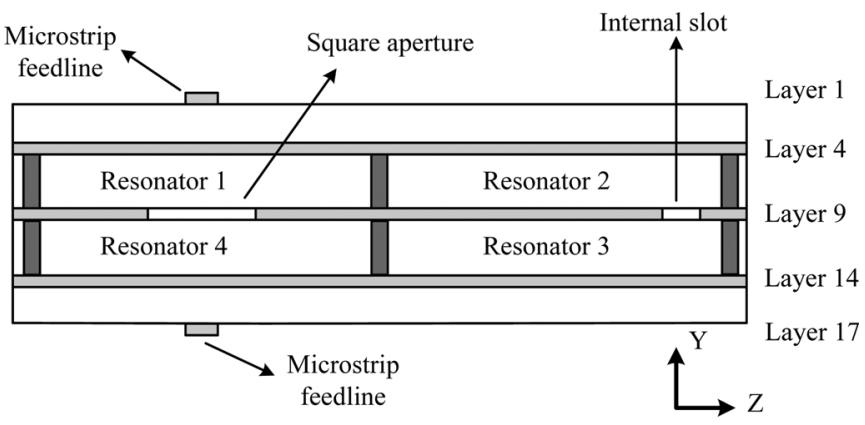

(b)

Fig. 1. Structure of a four-pole quasi-elliptic waveguide filter in a multilayer configuration. (a) 3-D overview. (b) Side view.

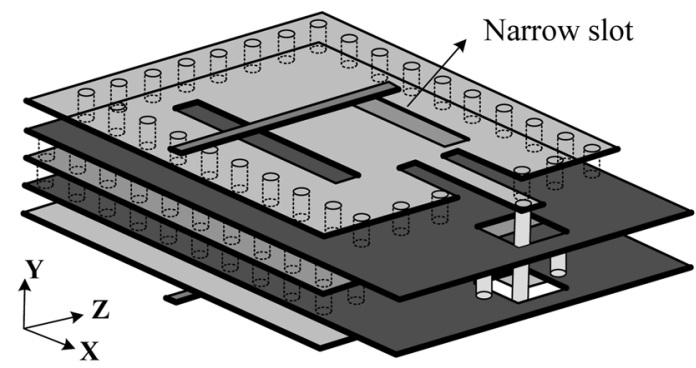

(a)

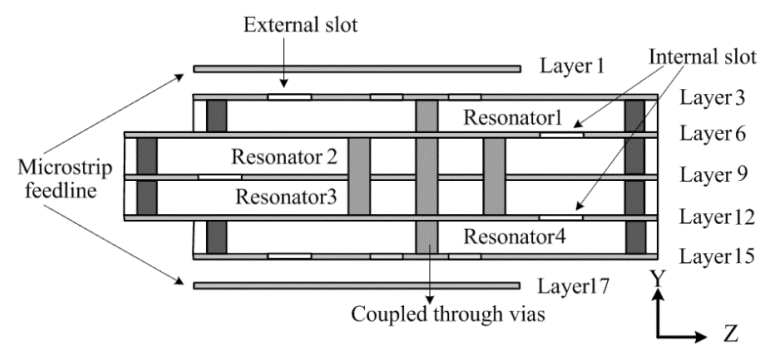

(b)

Fig. 2. Structure of a vertically stacked quasi-elliptic waveguide filter. (a) 3-D overview. (b) Side view.

need to be out of phase. However, it does not matter which one is positive or negative, as long as their signs are opposite.

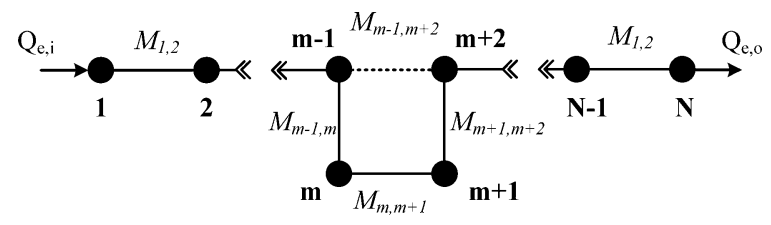

Fig. 3. General coupling structure of a quasi-elliptic filter.

The design parameters of bandpass filters, i.e., the coupling coefficients and the external $Q$ factor in Fig. 3, can be determined in terms of the circuit elements of a low-pass prototype filter [13].

After determining the required coupling coefficients and external $Q$ factor, the relationship between coupling coefficients and physical structures of coupled resonators should be established in order to determine the physical dimensions of the filter against the design parameters. The coupling coefficients of coupled resonators can be specified by two split resonate frequencies resulting from electromagnetic coupling [14], i.e.,

$$
M_{i, j}= \pm \frac{f_{p 2}^{2}-f_{p 1}^{2}}{f_{p 2}^{2}+f_{p 1}^{2}}
$$

In (1), $f_{p 1}$ and $f_{p 2}$ are defined to be the lower and higher resonance frequencies, respectively. The sign of the coupling coefficient is dependent on the physical structure of the coupled resonators. For filter design, the meaning of positive or negative coupling is rather relative. The positive and negative coupling will have an opposite phase response, which can be found by the $S$-parameter of the coupling structure.

The external $Q$ factor can be characterized by [13]

$$
Q_{e}=\frac{f_{0}}{\delta f_{3 \mathrm{~dB}}}
$$

where $f_{0}$ and $\delta f_{3} \mathrm{~dB}$ represent the resonance frequency and the $3-\mathrm{dB}$ bandwidth of the input or output resonator. By (1) and (2), design curves of the coupling coefficients and external $Q$ factor versus physical dimensions of coupled resonators can be established. The sizes of coupling structures are also obtained according to the design parameters.

\section{REALIZATION OF COUPLING COEFFICIENTS}

\section{A. LTCC Cavity Resonator}

The cavity resonator is formed by several stacked dielectric substrate with metal surfaces at the outer layers and via arrays as vertical sidewalls, which is shown in Fig. 4. The resonant frequencies of the cavity with a perfectly conducting wall can be obtained by [15]

$$
f_{m n l}=\frac{c}{2 \pi \sqrt{\varepsilon_{r}}} \sqrt{\left(\frac{m \pi}{a}\right)^{2}+\left(\frac{n \pi}{b}\right)^{2}+\left(\frac{l \pi}{d}\right)^{2}}
$$

where $\varepsilon_{r}$ is the relative dielectric constant, $c$ is the speed of light, $a, b$, and $d$ are the width, height, and length of the cavity, respectively, and $m, n$, and $l$ are the indices of the resonant mode. By (3), the initial dimensions of the synthesized waveguide cavity can be determined, and the final values are optimized by the 


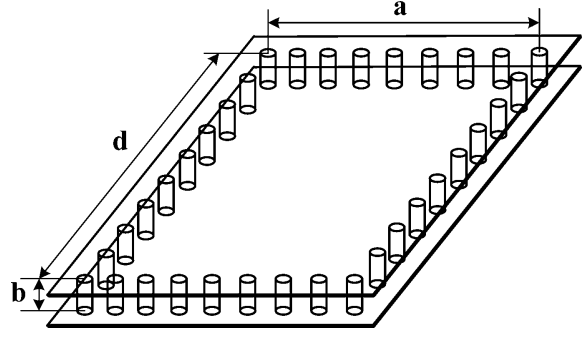

Fig. 4. Cavity resonator with metallic plates and via arrays.

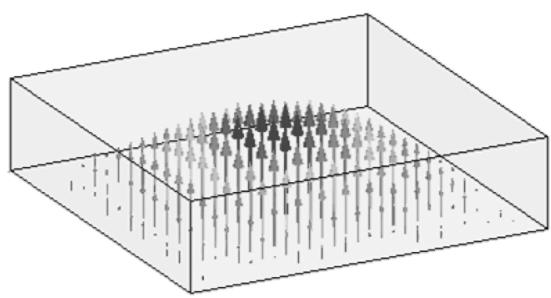

(a)

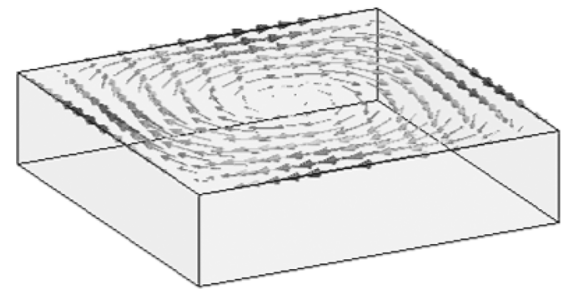

(b)

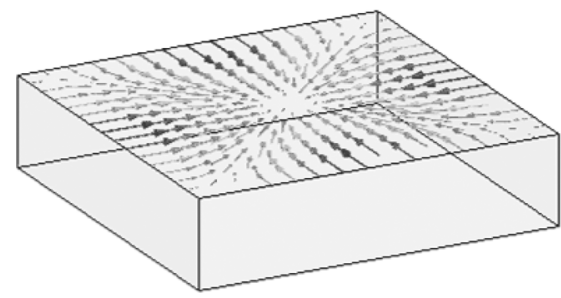

(c)

Fig. 5. Field patterns of $\mathrm{TE}_{101}$ mode of a single rectangular cavity. (a) Electric field. (b) Magnetic field. (c) Surface current on the metal plane.

eigenmode solution solver of a full-wave simulator, e.g., the High Frequency Structure Simulator (HFSS). Besides, the $Q$ factor of a cavity resonator increases with the cavity height. To get a higher $Q$ cavity, more substrate layers will be used to form a synthesized cavity according to the fabrication limitation.

The cavity resonators in Figs. 1 and 2 operate in their fundamental mode $\mathrm{TE}_{101}$ at a common center frequency. Fig. 5 shows the field patterns. The electric field is mainly concentrated at the center of the cavity and in the direction normal to the metal plane. The magnetic field is tangential to the metallic walls and rotates in the cavity. The magnetic field increases its strength gradually when approaching the sidewalls. The surface current will flow into the center of the metal plate in a radial shape.

Next, several structures of coupled resonators in LTCC technology are introduced. The cavities in the same layer are coupled by an inductive window, while the cavities between different layers are coupled by a square aperture in the center of

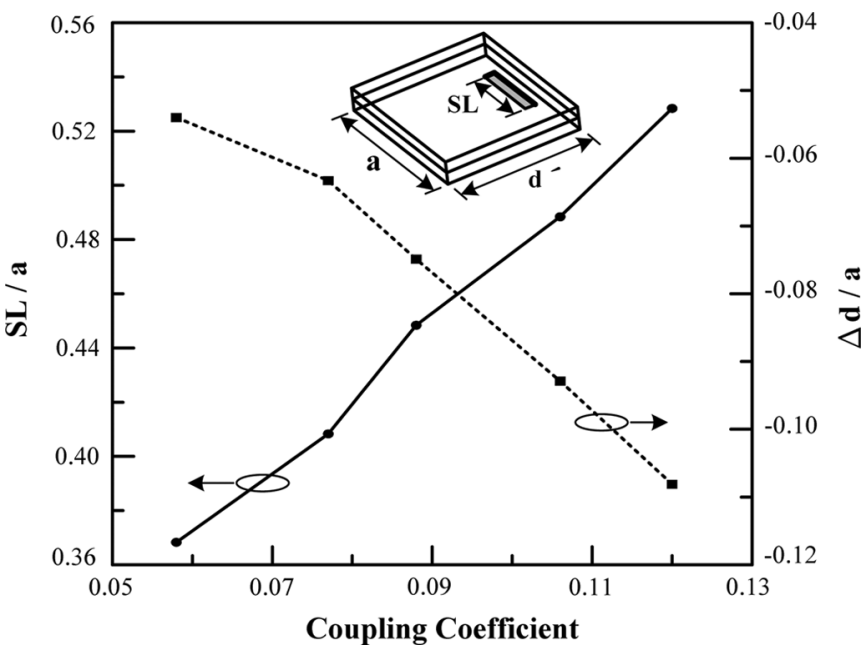

Fig. 6. Coupling coefficients of the slot coupling structure.

the common wall, a narrow slot at the edge of the common wall, or an additional thru via.

\section{B. Magnetic Coupling by Broad-Wall Slots}

To efficiently couple two adjacent cavities in different layers, a narrow slot in the common intermediate wall is placed near the sidewalls of the cavity and in the direction perpendicular to the surface current. It will significantly interrupt the surface current flow and introduce strong coupling, analogous to the design principle of waveguide slot antenna [16]. Hence, the coupling between adjacent cavities can be achieved by means of magnetic fields through the narrow slot in the common wall.

The coupling coefficient is affected by the length and position of the narrow slot. To get strong direct coupling, the narrow slot should be located as close to the sidewall of the cavity as possible. The coupling strength is then controlled by the slot length. Fig. 6 shows the relation between the slot length and coupling coefficients of the stacked cavities. As mentioned in Section II, the coupling coefficients are calculated by two split resonate frequencies, which can be obtained by an eigenmode solution solver of HFSS. Each cavity resonates at $31 \mathrm{GHz}$, with the cavity size $a \times b \times d=2.45 \times 0.25 \times 2.45 \mathrm{~mm}^{3}$ and the relative dielectric constant $\varepsilon_{r}=7.8$. According to fabrication limitation, the slot is located $0.2 \mathrm{~mm}$ from the cavity sidewall.

Due to the presence of the slot, the length of each cavity should be adjusted to compensate for the shifted resonant frequency [2]. $\Delta d$ will be the difference between the original cavity length $d$ and the modified cavity length $d /$ for the frequency compensation. The relation between the coupling coefficients and the variation of the cavity length is also plotted in Fig. 6. Both the adjustments in slot length and cavity length are normalized to the cavity width $a$ in Fig. 6 .

\section{Magnetic Coupling by Narrow-Wall Window}

The pair of vias composing the inductive window are used to control the coupling of the cavities at the same layer. It is a common coupling structure in the planar waveguide filter [3]. The coupling strength is controlled by the separation of the via pair. The wider the separation, the stronger the coupling can be. 


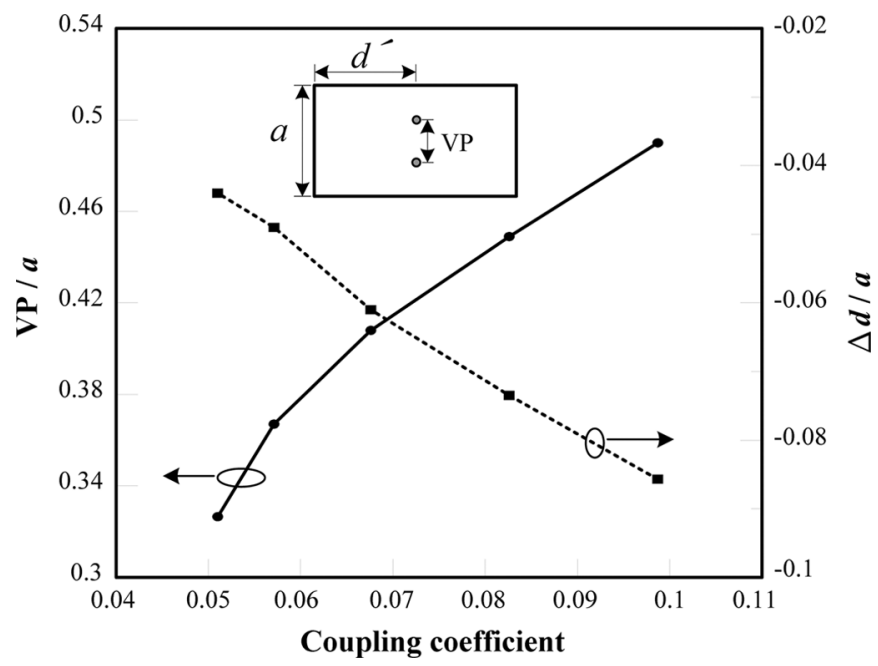

Fig. 7. Coupling coefficient of the inductive window coupling structure.

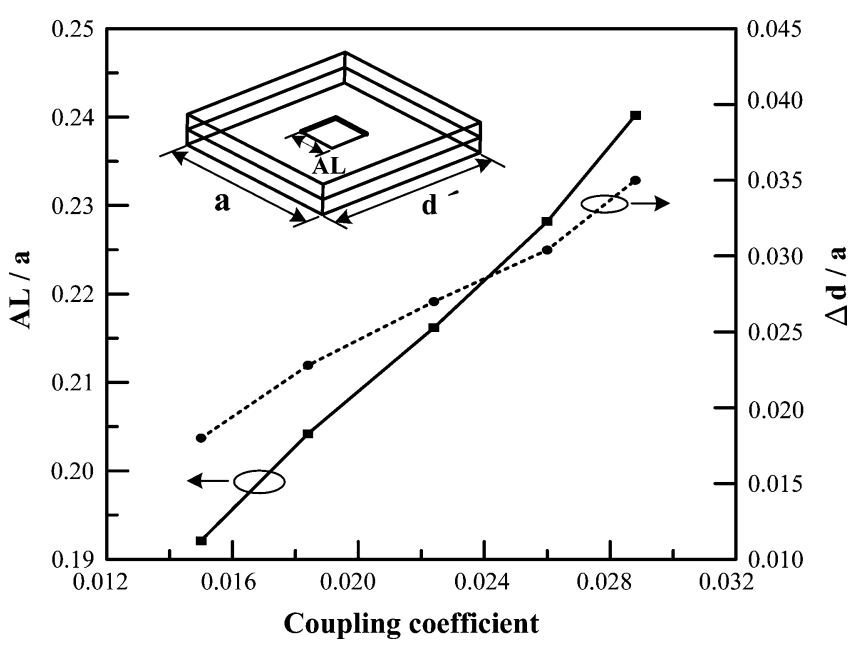

Fig. 8. Coupling coefficients of the square aperture coupling structure.

Fig. 7 shows the relation of coupling coefficients versus the via pitch (VP) and the cavity length variation $\Delta d$.

\section{Electric Coupling by Broad-Wall Aperture}

If a square aperture is opened at the center of two stacked cavities, where the electric field is a maximum, the coupling can be achieved in terms of an electric field normal to the aperture. Fig. 8 shows the aperture length (AL) and cavity length deviation $\Delta d$, which are both normalized to cavity width $a$, versus the coupling coefficients.

\section{E. Cross Coupling by Vias Connecting Nonadjacent Cavities}

As shown in Fig. 2, the first and last cavities are coupled by an additional through via, which provides the cross-coupling path to achieving a quasi-elliptic frequency response [17]. The crosscoupling structure is mainly formed by short-circuited CPW feed lines with a main thru-hole via and two shorter buried vias beside the main through via. The CPW feed lines are connected to the first and last resonators and the main thru-hole via is connected to the CPW feed lines.

In the LTCC process, the electric field of a grounded CPW is mainly concentrated under the signal line because of the small

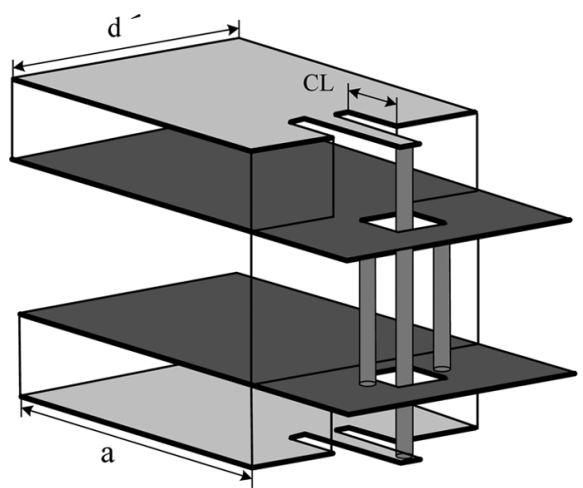

(a)

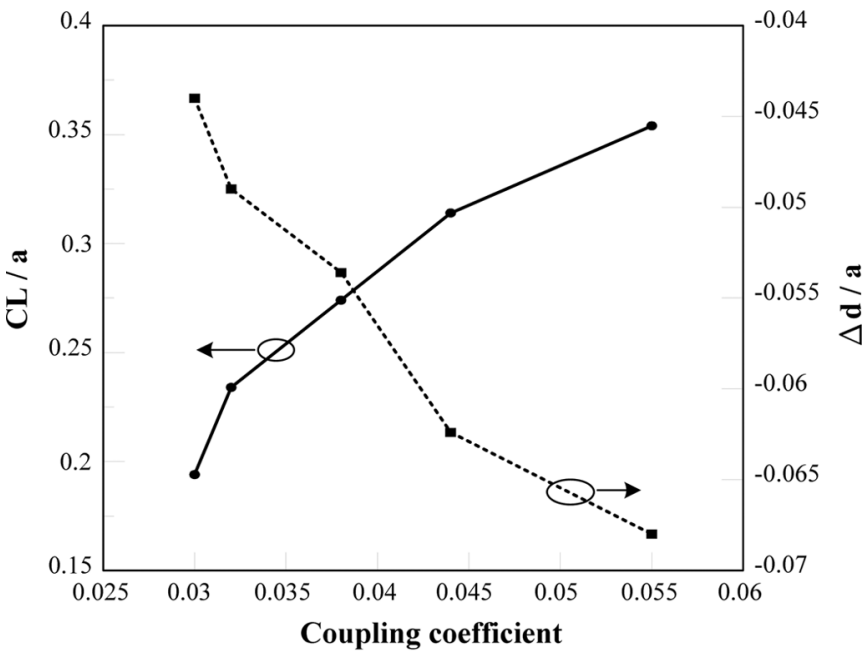

(b)

Fig. 9. Via coupling structure. (a) Overview. (b) Coupling coefficient.

substrate height and wide gap. The electric field distribution of a grounded $\mathrm{CPW}$ will be similar to that of $\mathrm{TE}_{101}$ of a cavity resonator and, therefore, energy can be gathered from the cavity easily with a CPW feed line. The energy will pass down along the main thru via and couple to the other cavity connected to the CPW feed line. Two shorter buried vias provide current return paths when energy is delivering.

The coupling coefficient between the first and last cavities can be extracted by a very weak excitation with the same method described in [2]. Two split resonant frequencies can be seen clearly from the $S$-parameter of the coupled resonators structure. The strength of the cross coupling can be controlled by the length of the CPW stretched into a cavity. When the short-circuited end of the CPW is closer to the center of the cavity where the electric field is strongest, more energy can be gathered from the resonator. The relation between the coupling coefficient and the CPW length is shown in Fig. 9. The coupling coefficient basically increases with the CPW length.

Fig. 10 plots the $S$-parameter of coupled resonators with a slot coupling structure and a via coupling structure. By comparing the phase responses in Fig. 10(a) and (b), it is clear that they are out-of-phase. That is to say, two extracted coupling coefficients have opposite signs [18]. Therefore, slot coupling and via coupling structures can be used to realize a quasi-elliptic filter. 


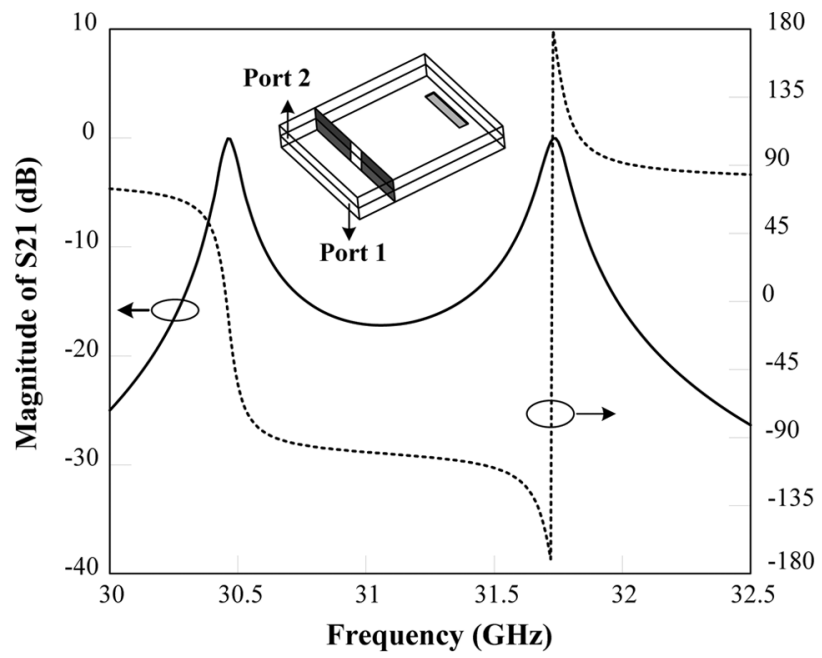

(a)

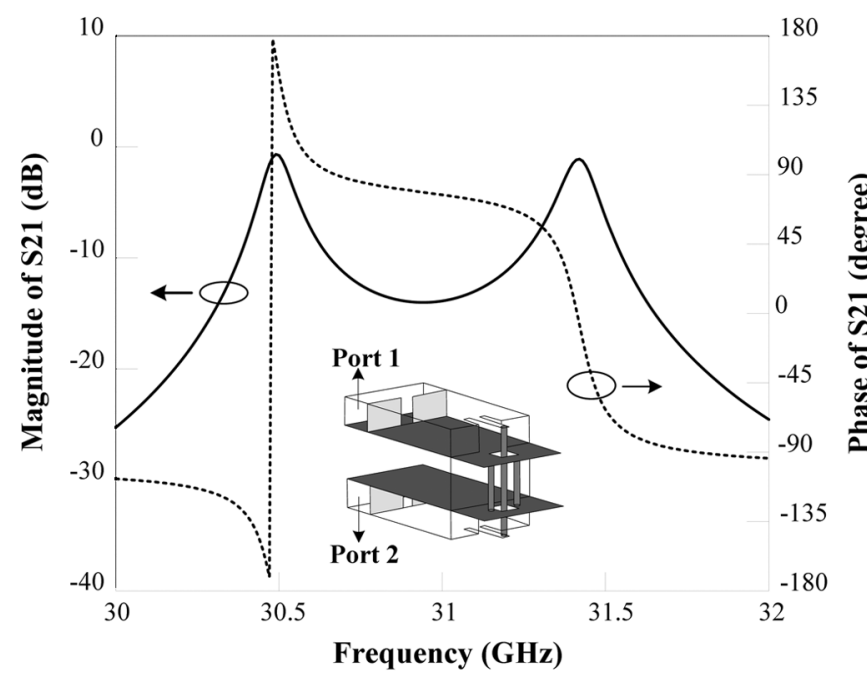

(b)

Fig. 10. Phase response of coupled resonators. (a) Slot coupling structure. (b) Via coupling structure.

\section{DESIGN EXAMPLES AND EXPERIMENTAL VERIFICATION}

Next, two kinds of cross coupling structures are utilized to realize the four-pole quasi-elliptic filters. When designing the filters, perfect conductor sidewalls are assumed for calculation efficiency. Based on the above procedure, the initial dimensions of the coupling slots, aperture, and inductive window are decided. The cavities sizes with small variation are also known. The entire filter structure is then optimized by HFSS to meet the design specification. After the initial design of the filter is accomplished, the metallic via arrays take the place of the perfect conductor sidewalls to complete the filter design.

\section{A. Feeding Structure}

The filter is excited by open-ended microstrip lines, as shown in Fig. 1. The slot discontinuity at the return path of the microstrip line causes strong coupling for the same reason that waveguide slot antenna radiates. To maximize the magnetic coupling, a virtual short is placed at the center of each slot by using a quarter-wavelength open stub beyond the slots center [19]. This kind of feeding structure can not only avoid dc power loss, but

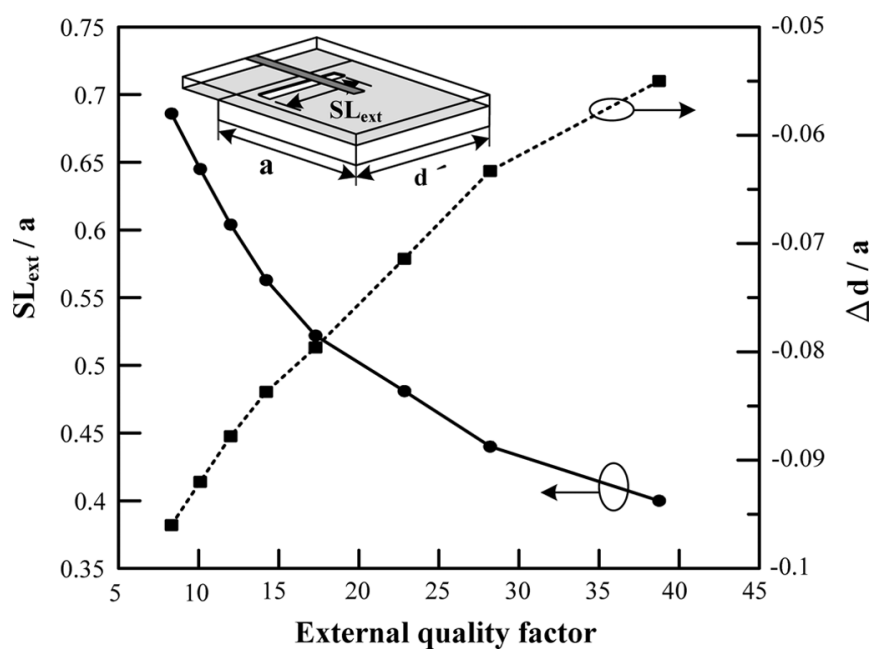

Fig. 11. External $Q$ factor of the microstrip line feeding structure.

also contribute to fabrication simplicity. The external $Q$ factor of the feeding structure is controlled by the external slot length and position. Fig. 11 shows the relation of the external $Q$ factor versus the slot length $\mathrm{SL}_{\text {ext }}$ and the cavity length variation $\Delta d$.

\section{B. Basic Stacked LTCC Filters Design}

A canonical waveguide filter with coupling between nonadjacent cavities can be utilized to achieve an elliptic-function filter response [20]. The cross couplings are achieved by a circle at the center of the common wall or by a narrow slot at the edge of the cavity. In the same concept, a quasi-elliptic filter realized by LTCC technology is presented here.

The configuration of the basic stacked LTCC filter is shown in Fig. 1. The coupling produced by means of electric and magnetic fields have opposite signs [21]; therefore, the filter architecture of Fig. 1 will conform to the general coupling structure in Fig. 3, which results in a quasi-elliptic frequency response.

The four-pole quasi-elliptic waveguide filter is designed and fabricated in LTCC. The specification of the filter is $10 \%$ fractional bandwidth centered at $31 \mathrm{GHz}$ with $20-\mathrm{dB}$ passband return loss. The element values of the low-pass prototype filter are found to be $g_{0}=1, g_{1}=0.95974, g_{2}=1.42192, J_{1}=$ -0.2183 , and $J_{2}=1.11769$. By [13], the coupling coefficients and I/O external $Q$ factor are

$$
\begin{aligned}
M & =\left[\begin{array}{cccc}
0 & 0.086 & 0 & -0.022 \\
0.086 & 0 & 0.079 & 0 \\
0 & 0.079 & 0 & 0.086 \\
-0.022 & 0 & 0.086 & 0
\end{array}\right] \\
Q_{e i} & =Q_{e o}=9.6 .
\end{aligned}
$$

The relative dielectric constant of the substrate is 7.8 and its loss tangent is 0.0078 at $30 \mathrm{GHz}$. The thickness of each metal layer is $13 \mu \mathrm{m}$ and the dielectric layer thickness between two metal layers is $50 \mu \mathrm{m}$. The cavity height is $250 \mu \mathrm{m}$, while the microstrip substrate height is $150 \mu \mathrm{m}$. The via diameter is $100 \mu \mathrm{m}$. To allow on-wafer measurement by coplanar probes, the input and output probe pad should be on the same layer. Therefore, a vertical transition composed of thru-hole vias is utilized to connect the bottom microstrip line to the top layer. Eight grounded 


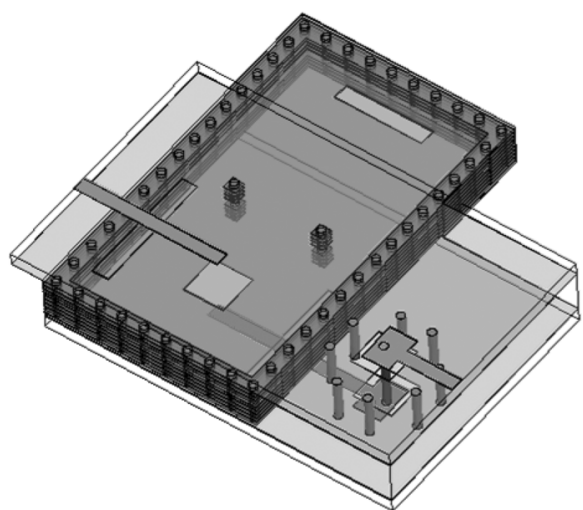

(a)

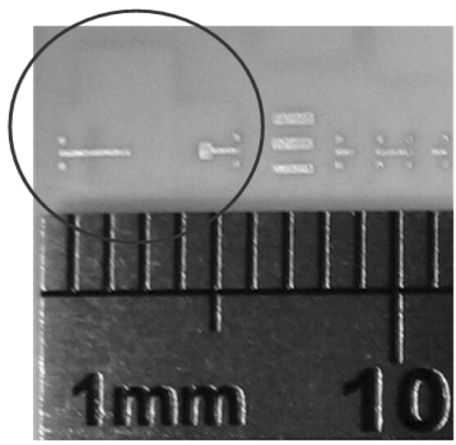

(b)

Fig. 12. (a) Hole structure of a four-pole quasi-elliptic waveguide filter with a vertical transition. (b) Fabricated filter.

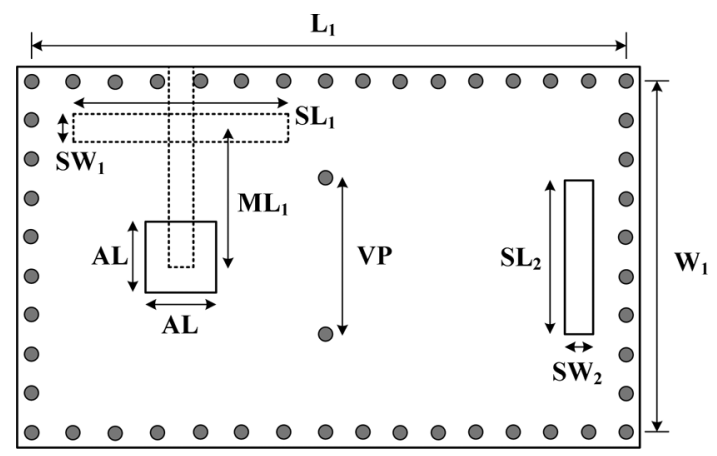

Fig. 13. Geometric parameters of the quasi-elliptic filter.

vias are located around the thru-hole via to mimic a coaxial transmission line effect.

Fig. 12(a) shows the whole filter configuration and Fig. 12(b) is a photograph of the fabricated filter. Geometric parameters of the filter are illustrated in Fig. 13 and summarized in Table I. The overall size of the four-pole quasi-elliptic LTCC waveguide filter without the vertical transition is $4.46 \times 2.72 \times 0.8 \mathrm{~mm}^{3}$, i.e., approximately $0.91 \lambda_{g} \times 0.55 \lambda_{g} \times 0.16 \lambda_{g}$, where $\lambda_{g}$ is the guided wavelength on the substrate at the center frequency. A full-wave simulator HFSS is used to calculate the $Q$ factor of a cavity. The $Q$ factor is found to be approximately 103 , which is similar to the measured data of approximately 99.

The frequency response of the filter is shown in Fig. 14, where the solid and dashed lines denote the measured and simulated results, respectively. The dashed-dotted lines represent the ideal
TABLE I

Geometric PARAmeters OF THE QUASI-ELLIPTIC FILTER

\begin{tabular}{|c|c|c|c|}
\hline Variable & Value $(u \mathrm{~m})$ & Variable & Value $(u \mathrm{~m})$ \\
\hline $\mathrm{L}_{1}$ & 4259 & $\mathrm{SL}_{1}$ & 1540 \\
\hline $\mathrm{W}_{1}$ & 2520 & $\mathrm{SW}_{1}$ & 200 \\
\hline $\mathrm{VP}_{1}$ & 1116 & $\mathrm{SL}_{2}$ & 1105 \\
\hline $\mathrm{AL}$ & 505 & $\mathrm{SW}_{2}$ & 200 \\
\hline $\mathrm{ML}_{1}$ & 1000 & & \\
\hline
\end{tabular}

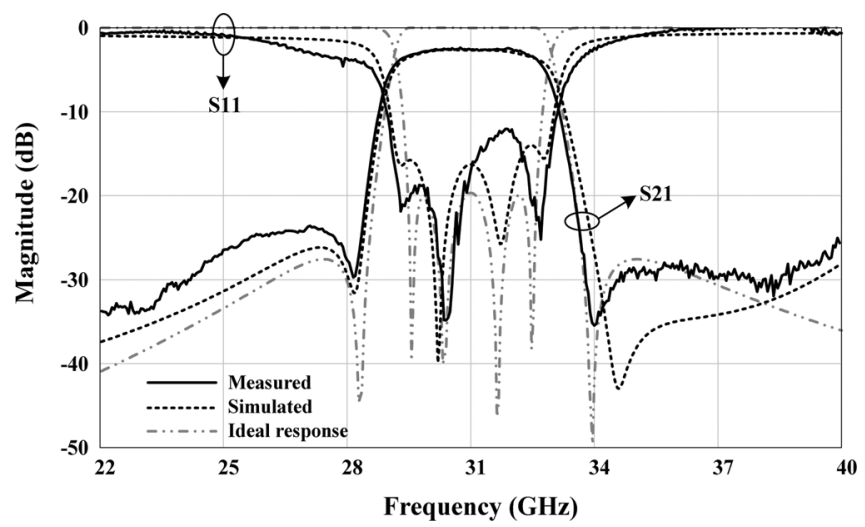

Fig. 14. Simulation and measurement results of the four-pole quasi-elliptic LTCC bandpass filter.

circuit response. The simulation result is not fully identical with the theoretical response. It can be contributed to the replacement of vertical sidewalls of the cavities by via arrays in LTCC and the vertical transitions for on-wafer measurement. When the perfect sidewalls are substituted by the via arrays, the major difference is the in-band return loss. This may be contributed to the variations in the coupling coefficients and external $Q$ factors, which make the frequency response deviated from that by the theoretical one. When the vertical transition is taken into consideration, The major discrepancy is the deterioration at the higher frequency side of the passband.

The measured center frequency of the filter is $30.9 \mathrm{GHz}$ and the $3-\mathrm{dB}$ bandwidth is $3.85 \mathrm{GHz}$. The passband insertion loss is approximately $2.55 \mathrm{~dB}$ and the passband return loss is greater than $12 \mathrm{~dB}$. Two attenuation poles near the cutoff frequencies of the passband can be clearly identified. The two attenuation poles are located at 28.2 and $34 \mathrm{GHz}$. The measured results are in good agreement with the full-wave simulation results by HFSS.

\section{Fully Stacked LTCC Filters Design}

The filter in Fig. 2 introduces a novel structure composed of vertically stacked cavities to realize a quasi-elliptic function filter. The configuration is re-plotted in Fig. 15, composed of four vertically stacked synthesized rectangular cavities. Adjacent cavities are coupled to each other by a narrow slot near the edge of the common wall. The cross-coupling path is realized by short-circuited CPW feed lines connected to the first and last resonators with a main thru-hole via connection.

A vertically stacked four-pole quasi-elliptic waveguide filter is designed and fabricated by the same LTCC process in the previous design example. The dimensions of other coupling slots 


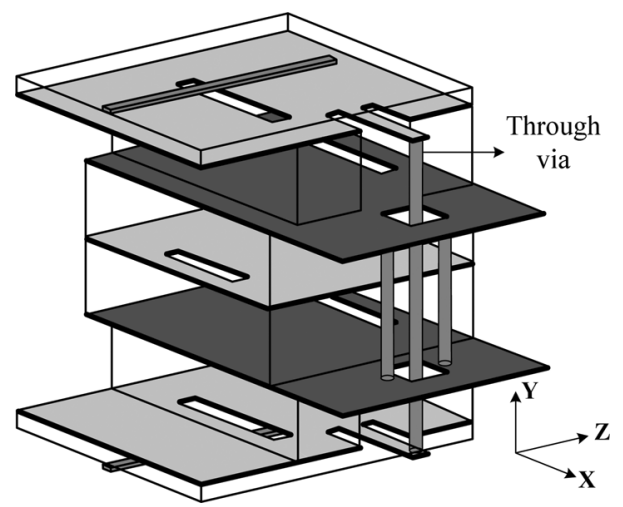

Fig. 15. Layer sketch of a fully stacked quasi-elliptic waveguide filter without via arrays.

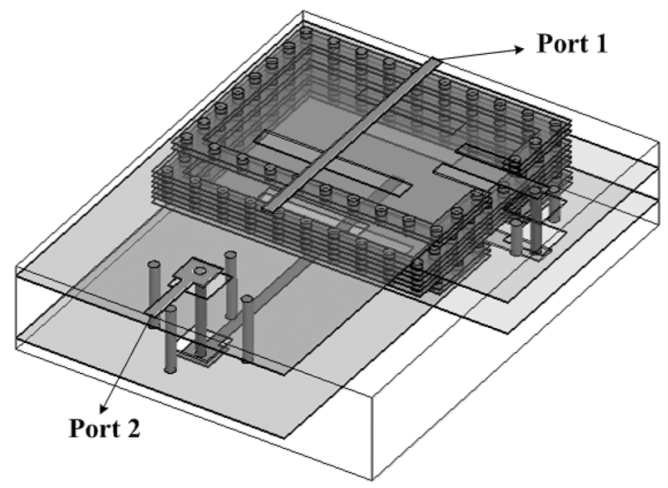

(a)

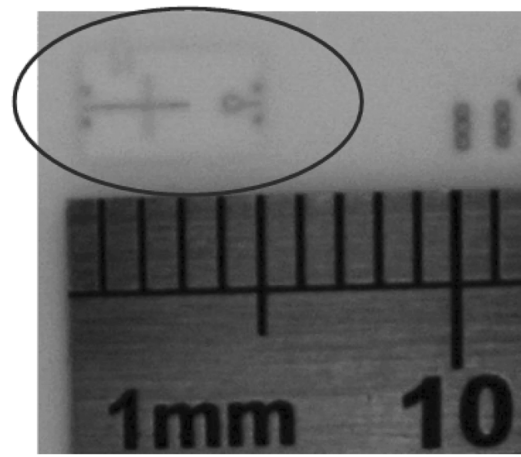

(b)

Fig. 16. (a) Entire structure of a fully stacked four-pole quasi-elliptic waveguide filter with a vertical transition. (b) Fabricated filter.

and cavities can be determined under the same design guide. Here, the specification of the filter is $10 \%$ fractional bandwidth centered at $30.2 \mathrm{GHz}$ with $20-\mathrm{dB}$ passband return loss. Same element values of the low-pass prototype filter in the previous example are used. The coupling coefficients and external $Q$ factor are equal to (4) because the same fractional bandwidth is chosen.

The whole filter configuration and the photograph of the fabricated filter are shown in Fig. 16. The cavity height is $150 \mu \mathrm{m}$. The microstrip line substrate height is $100 \mu \mathrm{m}$. To simplify the measurement, the input microstrip line at the top layer will feed the filter from the opposite direction. A vertical transition connecting microstrip lines at the top and bottom layers is used for on-wafer measurement, as mentioned in the previous example. Geometric parameters of the filter are illustrated in

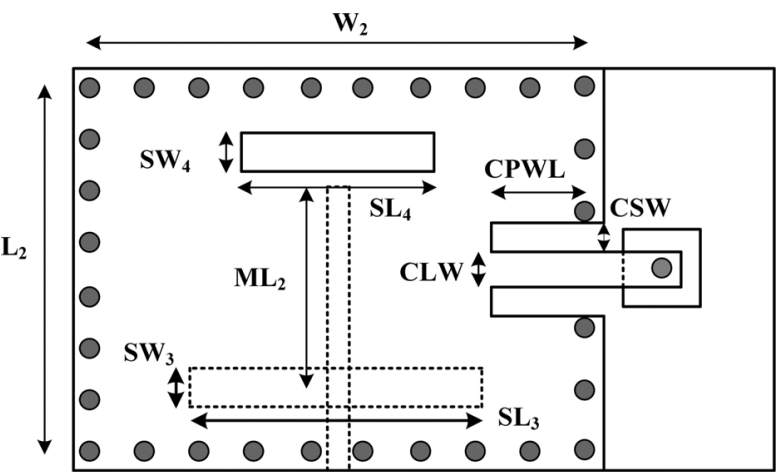

(a)

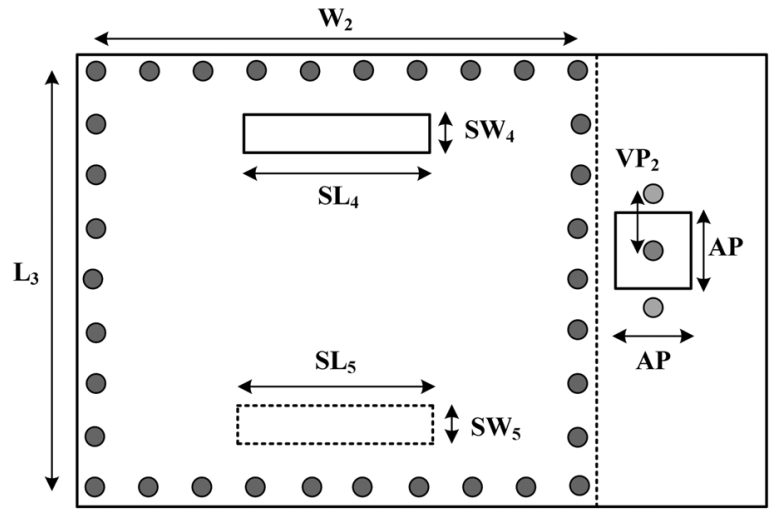

(b)

Fig. 17. Geometric parameters of the vertically stacked quasi-elliptic filter (a) Top view of the first and fourth cavity. (b) Top view of the second and third cavity.

TABLE II

GeOMETRIC PARAMETERS OF THE VerticAlLy STACKED QUASI-ELLIPTIC FILTER

\begin{tabular}{|c|c|c|c|}
\hline Variable & Value $(u \mathrm{~m})$ & Variable & Value $(u \mathrm{~m})$ \\
\hline $\mathrm{L}_{2}$ & 1890 & $\mathrm{SL}_{3}$ & 1520 \\
\hline $\mathrm{L}_{3}$ & 2200 & $\mathrm{SW}_{3}$ & 200 \\
\hline $\mathrm{W}_{2}$ & 2574 & $\mathrm{SL}_{4}$ & 1000 \\
\hline $\mathrm{AP}$ & 400 & $\mathrm{SW}_{4}$ & 200 \\
\hline $\mathrm{VP}_{2}$ & 300 & $\mathrm{SL}_{5}$ & 1040 \\
\hline $\mathrm{CLW}$ & 200 & $\mathrm{SW}_{5}$ & 200 \\
\hline $\mathrm{CSW}$ & 150 & $\mathrm{ML}_{2}$ & 1041 \\
\hline $\mathrm{CPWL}$ & 585 & & \\
\hline
\end{tabular}

Fig. 17 and summarized in Table II. The size of the vertically stacked four-pole quasi-elliptic LTCC waveguide filter without the vertical transition is $3.67 \times 2.4 \times 0.8 \mathrm{~mm}^{3}$, i.e., approximately $0.72 \lambda_{g} \times 0.47 \lambda_{g} \times 0.16 \lambda_{g}$.

The frequency response of the filter is shown in Fig. 18, where the solid and dashed lines denote measured and simulated results, respectively. The dashed-dotted lines represent the ideal circuit response. The measured center frequency of the filter is $29.5 \mathrm{GHz}$ and the $3-\mathrm{dB}$ bandwidth is $3.93 \mathrm{GHz}$. The passband insertion loss is approximately $2.8 \mathrm{~dB}$ and the passband return loss is greater than $12 \mathrm{~dB}$. The two attenuation poles are located at 26.85 and $33.05 \mathrm{GHz}$. The measured center frequency has down shifted approximately $2 \%$ as compared to the simulation 


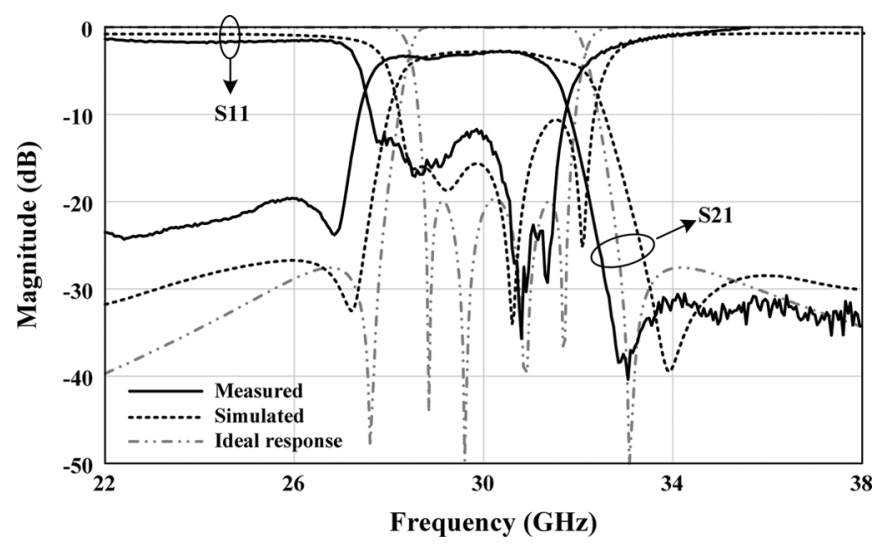

Fig. 18. Simulation and measurement results of the vertically stacked four-pole quasi-elliptic LTCC bandpass filter.

result. This may be contributed to the smaller LTCC shrinkage due to more metal plates used in this filter configuration. Therefore, the cavities are bigger than expected and will result in the down-shifted center frequency.

\section{CONCLUSION}

New structures have been proposed to realize the various coupling mechanisms required for quasi-elliptic bandpass filters design using stacked LTCC cavities. The idea has been validated by presenting two four-pole quasi-elliptic function bandpass filters in LTCC. Several coupling mechanisms between adjacent and nonadjacent resonators have been described in details. By this multilayer technology, the vertical coupling between cavities at different layers can be achieved and the filters will have compact size as compared to the conventional planar filters.

The filter with the electric field cross-coupling structure approximately occupies the size of two cavities, while the footprint of the filter with fully stacked cavities and cross-coupling via structure can achieve nearly $65 \%$ size reduction as compared to the conventional planar four-pole waveguide filters. The cross coupling between nonadjacent resonators is introduced to exhibit a single pair of transmission zeros near the passband at finite frequencies and, thus, much better selectivity. As a result, the proposed structures of the filters occupy a compact circuit area and have a good stopband response.

\section{ACKNOWLEDGMENT}

The authors would like to thank Dr. H.-H. Lin, C.-L. Wang, and C.-C. Chuang, all with the Computer and Communication Laboratory, Institute of Technology Industrial Research, Hsinchu, Taiwan, R.O.C., for their help in the fabrication and measurement of the LTCC filters.

\section{REFERENCES}

[1] H. Uchimura, T. Takenoshita, and M. Fujii, "Development of a laminated waveguide'," IEEE Trans. Microw. Theory Tech., vol. 46, no. 12, pp. 2437-2443, Dec. 1998.
[2] M. Ito, K. Maruhashi, K. Ikuina, T. Hashiguchi, S. Iwanaga, and K. Ohata, "A 60-GHz-band planar dielectric waveguide filter for flip-chip modules," IEEE Trans. Microw. Theory Tech., vol. 49, no. 12, pp. 2431-2436, Dec. 2001.

[3] D. Deslands and K. Wu, "Single-substrate integration technique of planar circuits and waveguide filters," IEEE Trans. Microw. Theory Tech., vol. 51, no. 2, pp. 593-596, Feb. 2003.

[4] D. Stephens, P. R. Young, and I. D. Robertson, "Millimeter-wave substrate integrated waveguides and filters in photoimageable thick-film technology," IEEE Trans. Microw. Theory Tech., vol. 53, no. 12, pp. 3832-3838, Dec. 2005.

[5] J.-H. Lee, S. Pinel, J. Papapolymerou, J. Laskar, and M. M. Tentzeris, "Low-loss LTCC cavity filters using system-on-package technology at $60 \mathrm{GHz}$," IEEE Trans. Microw. Theory Tech., vol. 53, no. 12, pp. 3817-3824, Dec. 2005.

[6] J. Papapolymerou, J.-C. Cheng, J. East, and L. P. B. Katehi, "A micromachined high- $Q X$-band resonator," IEEE Microw. Guided Wave Lett., vol. 7, no. 6, pp. 168-170, Jun. 1997.

[7] Z. C. Hao, W. Hong, X. P. Chen, J. X. Chen, K. Wu, and T. J. Cui, "Multilayered substrate integrated waveguide (MSIW) elliptic filter," IEEE Microw. Wireless Compon. Lett., vol. 15, no. 2, pp. 95-97, Feb. 2005.

[8] J. A. Ruiz-Cruz, M. A. E. Sabbagh, K. A. Zaki, J. M. Rebollar, and Y. Zhang, "Canonical ridge waveguide filters in LTCC or metallic resonators," IEEE Trans. Microw. Theory Tech., vol. 53, no. 1, pp. 174-182, Jan. 2005.

[9] M. M. Fahmi, J. A. Ruiz-Cruz, K. A. Zaki, and A. J. Piloto, "LTCC wideband canonical ridge waveguide filters," in IEEE MTT-S Int. Microw. Symp. Dig., 2005, pp. 249-252.

[10] R. Levy and S. B. Chon, "A history of microwave filter research, design, and development," IEEE Trans. Microw. Theory Tech., vol. MTT-32, no. 9, pp. 1055-1067, Sep. 1984.

[11] J. A. R. Cruz, K. A. Zaki, J. R. M. Garai, and J. M. Rebollar, "Rectangular waveguide elliptic filters with capacitive and inductive irises and integrated coaxial excitation," in IEEE MTT-S Int. Microw. Symp. Dig., Jun. 2005, pp. 269-272.

[12] J. S. Hong and M. J. Lancaster, "Transmission line filters with advanced filtering characteristics," in IEEE MTT-S Int. Microw. Symp. Dig., Jun. 2000, pp. 319-322.

[13] J. S. Hong and M. J. Lancaster, "Design of highly selective microstrip bandpass filters with a single pair of attenuation poles at finite frequencies," IEEE Trans. Microw. Theory Tech., vol. 48, no. 7, pp. 1098-1107, Jul. 2000.

[14] J. S. Hong and M. J. Lancaster, "Couplings of microstrip square openloop resonator for cross-couple planar microwave filters," IEEE Trans. Microw. Theory Tech., vol. 44, no. 12, pp. 2099-2109, Dec. 1996.

[15] D. M. Pozar, Microwave Engineering, 2nd ed. New York: Wiley, 1998, ch. 6.

[16] R. S. Elliott, Antenna Theory and Design. New York: Wiley, 2003, ch. 3 .

[17] T.-M. Shen, T.-Y. Huang, C.-F. Chen, and R.-B. Wu, "Design of a vertically stacked waveguide filter with novel cross coupling structures in LTCC," in Asia-Pacific Microw. Conf. Dig., Dec. 2006, pp. 1161-1164.

[18] J. S. Hong and M. J. Lancaster, Microstrip Filter for RF/Microwave Application. New York: Wiley, 2001, ch. 8.5.

[19] M. J. Hill, J. Papapolymerou, and R. W. Ziolkowski, "High- $Q$ micromachined resonant cavities in a $K$-band diplexer configuration," in Proc. Inst. Elect. Eng._Microw., Antennas, Propag., Oct. 2001, vol. 148 , no. 5 , pp. 307-312.

[20] A. E. Atia and A. E. Williams, "Nonminimum-phase optimum-amplitude bandpass waveguide filters," IEEE Trans. Microw. Theory Tech., vol. MTT-22, no. 4, pp. 425-431, Apr. 1976.

[21] T. Shen, H.-T. Shu, K. A. Zaki, and A. E. Atia, "Full-wave design of canonical waveguide filter by optimization," IEEE Trans. Microw. Theory Tech., vol. 51, no. 2, pp. 504-511, Feb. 2003.

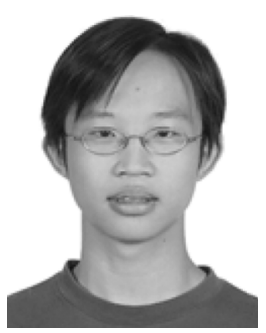

Tze-Min Shen was born in Chiayi, Taiwan, R.O.C., on August 5, 1981. He received the B.S. degree in electrical engineering and M.S. degree in communication engineering from National Taiwan University, Taipei, Taiwan, R.O.C., in 2004 and 2006, respectively, and is currently working toward the Ph.D. degree in communication engineering at National Taiwan University.

His research interests is the design of microwave filters. 


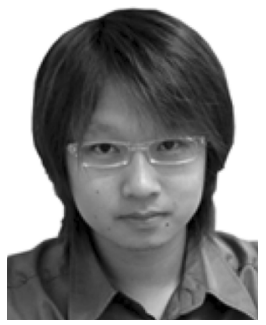

Chi-Feng Chen was born in PingTung, Taiwan, R.O.C., on September 3, 1979. He received the B.S. degree in physics from Chung Yuan Christian University, Taoyuan, Taiwan, R.O.C., in 2001, the M.S. degree in electrophysics from National Chiao Tung University, Hsinchu, Taiwan, R.O.C., in 2003, and the $\mathrm{Ph} . \mathrm{D}$. degree in communication engineering from National Taiwan University, Taipei, Taiwan, R.O.C., in 2006.

His research interests include the design of microwave filters and associated RF modules for microwave and millimeter-wave applications.

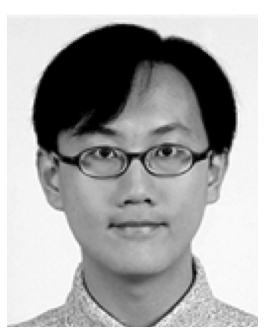

Ting-Yi Huang was born in Hualien, Taiwan, R.O.C., on November 12, 1977. He received the B.S. degree in electrical engineering and M.S. degree in communication engineering from National Taiwan University, Taipei, Taiwan, R.O.C., in 2000 and 2002 , respectively, and is currently working toward the Ph.D. degree in communication engineering at National Taiwan University.

His research interests include computational electromagnetics, the design of microwave filters, transitions, and associated RF modules for microwave and

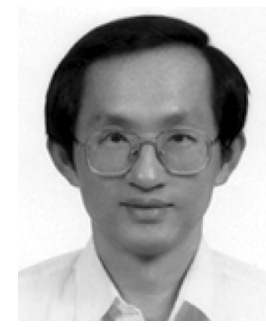

Ruey-Beei Wu (M'91-SM'97) was born in Tainan, Taiwan, R.O.C., on October 27, 1957. He received the B.S.E.E. and Ph.D. degrees from National Taiwan University, Taipei, Taiwan, R.O.C., in 1979 and 1985 , respectively.

In 1982, he joined the faculty of the Department of Electrical Engineering, National Taiwan University, where he is currently a Professor. He is also with the Graduate Institute of Communications Engineering, which was established in 1997. From March 1986 to February 1987, he was a Visiting Scholar with IBM East Fishkill, NY. From August 1994 to July 1995, he was with the Electrical Engineering Department, University of California at Los Angeles. He was appointed the Director of the National Center for High-Performance Computing from May 1998 to April 2000 and the Directorate General of Planning and Evaluation Division from November 2002 to July 2004, both under the National Science Council. Since August 2005, he has been Chairperson of the Department of Electrical Engineering, National Taiwan University. He has authored or coauthored over 150 papers in international journals or conferences. He served as an Associate Editor of the Journal of Chinese Institute of Electrical Engineering in 1996. His research interests include computational electromagnetics, transmission line and waveguide discontinuities, microwave and millimeter-wave planar circuits, and interconnection modeling for computer packaging.

Dr. Wu is a member Phi Tau Phi and the Chinese Institute of Electrical Engineers. He has been an associate editor for the IEEE TRANSACTIONS ON MicrowaVe THEORY AND TeCHNIQUES since 2005. He is an elected Executive Committee member of the IEEE Microwave Theory and Techniques Society (IEEE MTT-S) Taipei Chapter. He is an elected Executive Committee member of the Institute of United Radio Science (URSI) Taipei Section. He was the recipient of the Distinguished Research Award presented by the National Science Council (1990, 1993, 1995, and 1997) and the Outstanding Electrical Engineering Professor Award presented by the Chinese Institute of Electrical Engineers (1999). 